\title{
Publisher Correction: Directed evolution improves the catalytic efficiency of TEV protease
}

Mateo I. Sanchez and Alice Y. Ting (iD)

Correction to Nature Methods https://doi.org/10.1038/s41592-019-0665-7, published online 9 December 2019

In the version of this article originally published, there were errors in Figs. 3 and 4. In Fig. 3a, there was an extra delta symbol placed between the words 'TEV' and 'protease' in the label 'TEV protease library'. In Fig. 3f, the unit for $k_{\text {cat }} / K_{\mathrm{m}}$ was missing from the top rightmost column of the chart; it is ' $(\mathrm{s} \mathrm{mM})^{-1}$ '. In Fig. $4 \mathrm{~g}$, the labels in parentheses for the second and fourth 'GFP' rows should be 'SPARK expression' instead of 'FLARE expression. The errors have been corrected in the HTML and PDF versions of the paper.

Published online: 6 January 2020

https://doi.org/10.1038/s41592-019-0729-8

๑ The Author(s), under exclusive licence to Springer Nature America, Inc. 2020

\section{Publisher Correction: Orchestrating single-cell analysis with Bioconductor}

Robert A. Amezquita (D), Aaron T. L. Lun, Etienne Becht, Vince J. Carey, Lindsay N. Carpp (D), Ludwig Geistlinger, Federico Marini (D), Kevin Rue-Albrecht (D), Davide Risso, Charlotte Soneson (D), Levi Waldron (D), Hervé Pagès, Mike L. Smith D, Wolfgang Huber, Martin Morgan, Raphael Gottardo and Stephanie C. Hicks (D)

Correction to: Nature Methods https://doi.org/10.1038/s41592-019-0654-x, published online 2 December 2019.

In the version of this article initially published online, an author name was given as Federico Martini; the correct name is Federico Marini. The error has been corrected in the print, PDF and HTML versions of the article.

Published online: 11 December 2019

https://doi.org/10.1038/s41592-019-0700-8

(c) The Author(s), under exclusive licence to Springer Nature America, Inc. 2019

\section{Publisher Correction: Amplification-free single-cell whole-genome sequencing gets a makeover}

Vesna Todorovic

Correction to: Nature Methods https://doi.org/10.1038/s41592-019-0722-2, published online 6 January 2020.

In the version of this article initially published, work at the BC Cancer Research Centre was attributed to Samuel Aparicio. This has been changed to Samuel Aparicio and Sohrab P. Shah. The research team's description later in the paragraph has been changed from "now also including Sohrab P. Shah from the Memorial Sloan Kettering Cancer Center in New York" to "with Shah now at Memorial Sloan Kettering Cancer Center in New York." The errors have been corrected in the HTML and PDF versions of the article.

Published online: 22 January 2020

https://doi.org/10.1038/s41592-020-0739-6

(C) Springer Nature America, Inc. 2020 